\title{
On dimensionally restricted maps
}

\author{
by
}

\section{H. Murat Tuncali and Vesko Valov (North Bay)}

\begin{abstract}
Let $f: X \rightarrow Y$ be a closed $n$-dimensional surjective map of metrizable spaces. It is shown that if $Y$ is a $C$-space, then: (1) the set of all maps $g: X \rightarrow \mathbb{I}^{n}$ with $\operatorname{dim}(f \triangle g)=0$ is uniformly dense in $C\left(X, \mathbb{I}^{n}\right)$; (2) for every $0 \leq k \leq n-1$ there exists an $F_{\sigma}$-subset $A_{k}$ of $X$ such that $\operatorname{dim} A_{k} \leq k$ and the restriction $f \mid\left(X \backslash A_{k}\right)$ is $(n-k-1)$-dimensional. These are extensions of theorems by Pasynkov and Toruńczyk, respectively, obtained for finite-dimensional spaces. A generalization of a result due to Dranishnikov and Uspenskij about extensional dimension is also established.
\end{abstract}

1. Introduction. All spaces are assumed to be completely regular and all maps continuous. This paper is concerned with the following two results. The first one was proved by Pasynkov [25] (see [24] for noncompact versions) and the second one by Torunczyk [31]:

Theorem 1.1 (Pasynkov). Let $f: X \rightarrow Y$ be an $n$-dimensional map with $X$ and $Y$ being finite-dimensional compact metric spaces. Then there exists $g: X \rightarrow \mathbb{I}^{n}$ such that $f \triangle g: X \rightarrow Y \times \mathbb{I}^{n}$ is 0 -dimensional. Moreover, the set of all such $g$ is dense and $G_{\delta}$ in $C\left(X, \mathbb{I}^{n}\right)$ with respect to the uniform convergence topology.

Theorem 1.2 (Toruńczyk). Let $f: X \rightarrow Y$ be a $\sigma$-closed map of separable metric spaces with $\operatorname{dim} f=n$ and $\operatorname{dim} Y<\infty$. Then for each $0 \leq k \leq n-1$ there exists an $F_{\sigma}$-subset $A_{k}$ of $X$ such that $\operatorname{dim} A_{k} \leq k$ and the restriction $f \mid\left(X \backslash A_{k}\right)$ is $(n-k-1)$-dimensional.

The above two theorems are equivalent in the realm of compact spaces (see [19] and [29]). However, the problem whether they hold without any dimensional restrictions on $Y$ is still open. Sternfeld and Levin made a significant progress in solving this problem. In 1995, Sternfeld [29] proved that

2000 Mathematics Subject Classification: Primary 54F45; Secondary 55M10, 54C65.

$K e y$ words and phrases: finite-dimensional maps, extensional dimension, $C$-space.

The first author was partially supported by NSERC grant. Grant.

The second author was partially supported by Nipissing University Research Council 
if $f: X \rightarrow Y$ is an $n$-dimensional map between compact metric spaces, then $\operatorname{dim}(f \Delta g) \leq 1$ for almost all $g \in C\left(X, \mathbb{I}^{n}\right)$, where $f \triangle g$ denotes the diagonal product of the maps $f$ and $g$; equivalently, there exists a $\sigma$-compact $(n-1)$-dimensional subset $A$ of $X$ such that $\operatorname{dim}(f \mid(X \backslash A)) \leq 1$. Levin [19] improved Sternfeld's result showing that $\operatorname{dim}(f \triangle g) \leq 0$ for almost all maps $g \in C\left(X, \mathbb{I}^{n+1}\right)$, which is equivalent to the existence of a $\sigma$-compact $n$-dimensional set $A \subset X$ with $\operatorname{dim}(f \mid(X \backslash A)) \leq 0$.

In the present paper we generalize Theorems 1.1 and 1.2 to arbitrary metrizable spaces by replacing the finite dimensionality of $Y$ with the less restrictive condition of being a $C$-space. Recall that a space $X$ is a $C$-space [1] if for any sequence $\left\{\omega_{n}: n \in \mathbb{N}\right\}$ of open covers of $X$ there exists a sequence $\left\{\gamma_{n}: n \in \mathbb{N}\right\}$ of open disjoint families in $X$ such that each $\gamma_{n}$ refines $\omega_{n}$ and $\bigcup\left\{\gamma_{n}: n \in \mathbb{N}\right\}$ covers $X$. The $C$-space property was introduced by Haver [15] for compact metric spaces, and Addis and Gresham [1] extended Haver's definition to more general spaces. All countable-dimensional metrizable spaces (spaces which are countable unions of finite-dimensional subsets), in particular all finite-dimensional ones, form a proper subclass of the class of $C$-spaces because there exists a metric $C$-compactum which is not countable-dimensional [27].

Here is a generalized version of Theorem 1.1.

TheOREM 1.3. Let $f: X \rightarrow Y$ be a closed map of metric spaces with $\operatorname{dim} f=n$ and $Y$ a $C$-space. Then all maps $g: X \rightarrow \mathbb{I}^{n}$ such that $\operatorname{dim}(f \triangle g)$ $=0$ form a dense subset of $C\left(X, \mathbb{I}^{n}\right)$ with respect to the uniform convergence topology. Moreover, if $f$ is $\sigma$-perfect, then this set is dense and $G_{\delta}$ in $C\left(X, \mathbb{I}^{n}\right)$ with respect to the source limitation topology.

Theorem 1.3 answers affirmatively Pasynkov's question in [25] whether Theorem 1.1 is true for countable-dimensional spaces.

For any map $f: X \rightarrow Y, \operatorname{dim} f=\sup \left\{\operatorname{dim} f^{-1}(y): y \in Y\right\}$ is the dimension of $f$. We say that a surjective map $f: X \rightarrow Y$ is $\sigma$-closed (resp., $\sigma$-perfect) if $X$ is the union of countably many closed sets $X_{i}$ such that each restriction $f \mid X_{i}: X_{i} \rightarrow f\left(X_{i}\right)$ is a closed (resp., perfect) map and all $f\left(X_{i}\right)$ are closed in $Y$.

Using Theorem 1.3 we prove the following generalization of Theorem 1.2:

TheOREM 1.4. Let $f: X \rightarrow Y$ be a $\sigma$-closed map of metric spaces with $\operatorname{dim} f=n$ and $Y$ a $C$-space. Then for each $0 \leq k \leq n-1$ there exists an $F_{\sigma}$-subset $A_{k}$ of $X$ such that $\operatorname{dim} A_{k} \leq k$ and $f \mid\left(X \backslash A_{k}\right)$ is $(n-k-1)$ dimensional.

A few words about this note. In Section 2 we give a characterization of finite-dimensional proper maps (see Theorem 2.2), which is the main tool in the proof of Theorem 1.3. The proof of Theorem 2.2 is based on 
a selection theorem established by V. Gutev and the second author [14, Theorem 1.1]. Sections 3 and 4 are devoted to the proof of Theorems 1.3 and 1.4, respectively. In the last Section 5 we provide applications of the main results. One of them is a generalization of a result by Dranishnikov and Uspenskij [10] concerning maps which lower extensional dimension, another one is a parametric version of the Bogaty $\breve{1}$ representation theorem for $n$-dimensional metrizable spaces [2]. Some results in the spirit of Pasynkov's recent paper [24] are also obtained.

We wish to thank the referee who recommended some improvements of the paper.

2. Finite-dimensional maps. In this section we provide a characterization of $n$-dimensional perfect maps onto paracompact $C$-spaces (see Theorem 2.2 below).

For any spaces $M$ and $K$, we denote by $C(K, M)$ the set of all continuous maps from $K$ into $M$. If $(M, d)$ is a metric space and $K$ is any space, then the source limitation topology on $C(K, M)$ is defined in the following way: a subset $U \subset C(K, M)$ is open in $C(K, M)$ with respect to the source limitation topology provided for every $g \in U$ there exists a continuous function $\alpha: K \rightarrow(0, \infty)$ such that $\bar{B}(g, \alpha) \subset U$. Here, $\bar{B}(g, \alpha)$ denotes the set $\{h \in C(K, M): d(g(x), h(x)) \leq \alpha(x)$ for each $x \in K\}$.

The source limitation topology is also known as the fine topology and $C(K, M)$ with this topology has the Baire property provided $(M, d)$ is a complete metric space [23]. We also need the following fact: if $K$ is paracompact and $F \subset K$ closed, then the restriction map $p_{F}: C(K, M) \rightarrow C(F, M)$, $p_{F}(g)=g \mid F$, is continuous when both $C(K, M)$ and $C(F, M)$ are equipped with the source limitation topology; moreover $p_{F}$ is open and surjective provided $M$ is a closed convex subset of a Banach space and $d$ is the metric on $M$ generated by the norm. Finally, when $K$ and $M$ are metrizable, the source limitation topology on $C(K, M)$ does not depend on the metric on $M$.

Let $\omega$ be an open cover of the space $M$ and $m \in \mathbb{N} \cup\{0\}$. A family $\gamma$ of subsets of $M$ is said to be $(m, \omega)$-discrete in $M$ if $\operatorname{ord}(\gamma) \leq m+1$ (i.e., every point of $M$ belongs to at most $m+1$ elements of $\gamma$ ) and $\gamma$ refines $\omega$; a subset of $M$ which can be represented as the union of an open $(m, \omega)$-discrete family in $M$ is called $(m, \omega)$-discrete; a map $g: M \rightarrow Z$ is $(m, \omega)$-discrete if every $z \in g(M)$ has a neighborhood $V_{z}$ in $Z$ such that $g^{-1}\left(V_{z}\right)$ is $(m, \omega)$-discrete in $M$.

We denote by $\operatorname{cov}(M)$ the family of all open covers of $M$. In case $(M, d)$ is a metric space, $B_{\varepsilon}(x)$ (resp., $\left.\bar{B}_{\varepsilon}(x)\right)$ stands for the open (resp., closed) ball in $(M, d)$ with center $x$ and radius $\varepsilon$. 
Lemma 2.1. If $\omega \in \operatorname{cov}(M)$ and $K \subset M$ is compact, then every functionally open $(m, \omega)$-discrete subset of $K$ can be extended to an $(m, \omega)$ discrete subset of $M$.

Proof. Let $U \subset K$ be functionally open and $(m, \omega)$-discrete in $K$ and $\gamma=\left\{U_{s}: s \in A\right\}$ an open $(m, \omega)$-discrete family in $K$ whose union is $U$. Since $U$ is paracompact (being functionally open in $K$ ), we can suppose that $\gamma$ is locally finite and there exists a partition of unity $\left\{f_{s}: s \in A\right\}$ in $U$ such that $U_{s}=f_{s}^{-1}((0,1])$ for each $s \in A$. Denote by $\mathcal{N}$ the nerve of $\gamma$ with the Whitehead topology and define $f: U \rightarrow \mathcal{N}$ by $f(x)=\sum_{s \in A} f_{s}(x) s$. Observe that $\mathcal{N}$ is at most $m$-dimensional because $\operatorname{ord}(\gamma) \leq m+1$. Let $W$ be a functionally open subset of $\beta M$ with $W \cap K=U$. Then, by [11], there exists an open set $V \subset W$ containing $U$ and an extension $g: V \rightarrow \mathcal{N}$ of $f$. The map $g$ generates maps $g_{s}: V \rightarrow[0,1]$ such that each $g_{s}$ extends $f_{s}$. We finally choose $G_{s} \in \omega$ with $U_{s} \subset G_{s}, s \in A$, and define $V_{s}=G_{s} \cap g_{s}^{-1}((0,1])$. Then the family $\left\{V_{s}: s \in A\right\}$ is $(m, \omega)$-discrete in $M$ and $\bigcup_{s \in A} V_{s}$ is the required $(m, \omega)$-discrete extension of $U$.

Throughout the paper $\mathbb{I}^{k}$ denotes the $k$-dimensional cube equipped with the Euclidean metric $d_{k}$, and $D_{k}$ denotes the uniform convergence metric on $C\left(X, \mathbb{I}^{k}\right)$ generated by $d_{k}$. If $f: X \rightarrow Y$, we denote by $C\left(X, Y \times \mathbb{I}^{k}, f\right)$ the set of all maps $h: X \rightarrow Y \times \mathbb{I}^{k}$ such that $\pi_{Y} \circ h=f$, where $\pi_{Y}$ : $Y \times \mathbb{I}^{k} \rightarrow Y$ is the projection. For any $\omega \in \operatorname{cov}(X)$ and closed $K \subset X$, $C_{(m, \omega)}\left(X \mid K, Y \times \mathbb{I}^{k}, f\right)$ stands for the set of all $h \in C\left(X, Y \times \mathbb{I}^{k}, f\right)$ with $h \mid K$ being $(m, \omega)$-discrete (as a map from $K$ into $\left.Y \times \mathbb{I}^{k}\right)$ and $C_{(m, \omega)}\left(X \mid K, \mathbb{I}^{k}\right)$ consists of all $g \in C\left(X, \mathbb{I}^{k}\right)$ such that $f \triangle g \in C_{(m, \omega)}\left(X \mid K, Y \times \mathbb{I}^{k}, f\right)$. In case $K=X$ we write simply $C_{(m, \omega)}\left(X, Y \times \mathbb{I}^{k}, f\right)\left(\right.$ resp., $\left.C_{(m, \omega)}\left(X, \mathbb{I}^{k}\right)\right)$ instead of $C_{(m, \omega)}\left(X \mid X, Y \times \mathbb{I}^{k}, f\right)$ (resp., $\left.C_{(m, \omega)}\left(X \mid X, \mathbb{I}^{k}\right)\right)$.

Now we can establish the following characterization of $n$-dimensional perfect maps:

THEOREM 2.2. Let $f: X \rightarrow Y$ be a perfect surjection between paracompact spaces with $Y$ being a $C$-space. Then $\operatorname{dim} f \leq n$ if and only if for any $\omega \in \operatorname{cov}(X)$ and $0 \leq k \leq n$ the set $C_{(n-k, \omega)}\left(X, \mathbb{I}^{k}\right)$ is open and dense in $C\left(X, \mathbb{I}^{k}\right)$ with respect to the source limitation topology.

Sufficiency is a consequence of the following observation: if $C_{(0, \omega)}\left(X, \mathbb{I}^{n}\right)$ is not empty for all $\omega \in \operatorname{cov}(X)$, then every open cover of $f^{-1}(y), y \in Y$, admits an open refinement of order $\leq n+1$, i.e. $\operatorname{dim} f^{-1}(y) \leq n$. Indeed, let $\gamma$ be a family of open subsets of $X$ covering $f^{-1}(y)$. Then $\omega=$ $\gamma \cup\left\{X \backslash f^{-1}(y)\right\} \in \operatorname{cov}(X)$, so there exists $g \in C_{(0, \omega)}\left(X, \mathbb{I}^{n}\right)$. Obviously, $g \mid f^{-1}(y)$ is $(0, \omega)$-discrete. Hence, every $z \in H=g\left(f^{-1}(y)\right)$ has a neighborhood $G_{z}$ in $\mathbb{I}^{n}$ with $g^{-1}\left(G_{z}\right) \cap f^{-1}(y)$ being the union of a disjoint family $\mu_{z}$ of sets open in $f^{-1}(y)$ which refines $\omega$. Take finitely many $z(i) \in H$, 
$i=1, \ldots, p$, such that $\lambda=\left\{G_{z(i)}: i=1, \ldots, p\right\}$ covers $H$. Since $\operatorname{dim} H \leq n$, we can suppose that $\operatorname{ord}(\lambda) \leq n+1$. Then $\mu=\bigcup\left\{\mu_{z(i)}: i=1, \ldots, p\right\}$ is an open cover of $f^{-1}(y)$ refining $\gamma$ and $\operatorname{ord}(\mu) \leq n+1$.

To prove necessity we need a few lemmas; the proof will be completed by Lemma 2.9. In all these lemmas we suppose that $X$ and $Y$ are given paracompact spaces and $f: X \rightarrow Y$ a perfect surjective map with $\operatorname{dim} f \leq n$, where $n \in \mathbb{N}$. We also fix $\omega \in \operatorname{cov}(X)$, an integer $k$ such that $0 \leq k \leq n$, and an arbitrary $m \in \mathbb{N} \cup\{0\}$.

Lemma 2.3. Let $g \in C_{(m, \omega)}\left(X \mid f^{-1}(y), \mathbb{I}^{k}\right)$ for some $y \in Y$. Then there exists a neighborhood $U$ of $y$ in $Y$ such that $g \mid f^{-1}(U)$ is $(m, \omega)$-discrete.

Proof. Obviously, $g \in C_{(m, \omega)}\left(X \mid f^{-1}(y), \mathbb{I}^{k}\right)$ implies that $g \mid f^{-1}(y)$ is an $(m, \omega)$-discrete map. Hence, for every $x \in f^{-1}(y)$ there exists an open neighborhood $V_{g(x)}$ of $g(x)$ in $\mathbb{I}^{k}$ such that $g^{-1}\left(V_{g(x)}\right) \cap f^{-1}(y)$ is an $(m, \omega)$-discrete set in $f^{-1}(y)$. Since $V_{g(x)}$ is functionally open in $\mathbb{I}^{k}$, so is $g^{-1}\left(V_{g(x)}\right) \cap f^{-1}(y)$ in $f^{-1}(y)$. Then, by Lemma 2.1 , there is an $(m, \omega)$-discrete subset $W_{x}$ in $X$ extending $g^{-1}\left(V_{g(x)}\right) \cap f^{-1}(y)$. Therefore, for every $x \in f^{-1}(y)$ we have $(f \triangle g)^{-1}(f(x), g(x))=f^{-1}(y) \cap g^{-1}(g(x)) \subset W_{x}$ and, since $f \triangle g$ is a closed map, there exists an open neighborhood $H_{x}=U_{y}^{x} \times G_{x}$ of $(y, g(x))$ in $Y \times \mathbb{I}^{k}$ with $S_{x}=(f \triangle g)^{-1}\left(H_{x}\right) \subset W_{x}$. Next, choose finitely many points $x(i) \in f^{-1}(y), i=1, \ldots, p$, such that $f^{-1}(y) \subset \bigcup_{i=1}^{p} S_{x(i)}$. As $f$ is a closed map we can find a neighborhood $U_{y}$ of $y$ in $Y$ such that $U_{y} \subset \bigcap_{i=1}^{p} U_{y}^{x(i)}$ and $f^{-1}\left(U_{y}\right) \subset \bigcup_{i=1}^{p} S_{x(i)}$.

Let us show that $g \mid f^{-1}\left(U_{y}\right)$ is $(m, \omega)$-discrete. Indeed, if $z \in f^{-1}\left(U_{y}\right)$, then $z \in S_{x(j)}$ for some $j$ and $g(z) \in G_{x(j)}$ because $S_{x(j)}=f^{-1}\left(U_{y}^{x(j)}\right) \cap$ $g^{-1}\left(G_{x(j)}\right)$. Consequently, $f^{-1}\left(U_{y}\right) \cap g^{-1}\left(G_{x(j)}\right) \subset S_{x(j)} \subset W_{x(j)}$. Therefore, $G_{x(j)}$ is a neighborhood of $g(z)$ such that $f^{-1}\left(U_{y}\right) \cap g^{-1}\left(G_{x(j)}\right)$ is $(m, \omega)$ discrete in $f^{-1}\left(U_{y}\right)$ as a subset of the $(m, \omega)$-discrete set $W_{x(j)}$ in $X$.

Corollary 2.4. If $g \in C_{(m, \omega)}\left(X \mid f^{-1}(y), \mathbb{I}^{k}\right)$ for every $y \in Y$, then $g \in C_{(m, \omega)}\left(X, \mathbb{I}^{k}\right)$.

Proof. We need to show that $f \triangle g$ is $(m, \omega)$-discrete, i.e. for any $x \in X$ there exist neighborhoods $U_{y}$ of $y=f(x)$ in $Y$ and $G_{x}$ of $g(x)$ in $\mathbb{I}^{k}$ such that $f^{-1}\left(U_{y}\right) \cap g^{-1}\left(G_{x}\right)$ is $(m, \omega)$-discrete in $X$. Indeed, by Lemma 2.3 , there exists a neighborhood $U_{y}$ of $y$ in $Y$ such that $g \mid f^{-1}\left(U_{y}\right)$ is $(m, \omega)$-discrete. Therefore, we can find a neighborhood $G_{x}$ of $g(x)$ in $\mathbb{I}^{k}$ with $f^{-1}\left(U_{y}\right) \cap$ $g^{-1}\left(G_{x}\right)$ being $(m, \omega)$-discrete in $f^{-1}\left(U_{y}\right)$. Consequently, $f^{-1}\left(U_{y}\right) \cap g^{-1}\left(G_{x}\right)$ is $(m, \omega)$-discrete in $X$.

Lemma 2.5. The set $C_{(m, \omega)}\left(X \mid K, \mathbb{I}^{k}\right)$ is open in $C\left(X, \mathbb{I}^{k}\right)$ with respect to the source limitation topology for any closed $K \subset X$. 
Proof. Let $g_{0} \in C_{(m, \omega)}\left(X \mid K, \mathbb{I}^{k}\right)$. We are going to find $\alpha \in C(X,(0, \infty))$ with $\bar{B}\left(g_{0}, \alpha\right) \subset C_{(m, \omega)}\left(X \mid K, \mathbb{I}^{k}\right)$. Since each restriction $g_{0} \mid\left(f^{-1}(y) \cap K\right)$, $y \in H=f(K)$, is $(m, \omega)$-discrete, by Lemma 2.3 , for every $y \in H$ there exists a neighborhood $U_{y}$ of $y$ in $Y$ such that $g_{0} \mid\left(f^{-1}\left(U_{y}\right) \cap K\right)$ is $(m, \omega)$ discrete. Then $\omega_{1}=\left\{U_{y}: y \in H\right\} \cup\{Y \backslash H\}$ is an open cover of $Y$. As $Y$ is paracompact, we can find a metric space $(M, d)$, a surjection $p: Y \rightarrow M$ and $\mu \in \operatorname{cov}(M)$ such that $p^{-1}(\mu)$ refines $\omega_{1}$. Hence, every $z \in p(H)$ has a neighborhood $W_{z}$ in $M$ such that $g_{0} \mid(p \circ f)^{-1}\left(W_{z}\right) \cap K$ is $(m, \omega)$-discrete. The last condition implies that $h_{0} \mid K$ is $(m, \omega)$-discrete, where $h_{0}=(p \circ f) \triangle g_{0}$. Now we need the following:

Claim. There exists an open family $\gamma$ in $M \times \mathbb{I}^{k}$ covering $h_{0}(K)$ such that every $g \in C\left(X, \mathbb{I}^{k}\right)$ belongs to $C_{(m, \omega)}\left(X \mid K, \mathbb{I}^{k}\right)$ provided $h \mid K$ is $\gamma$-close to $h_{0} \mid K$, where $h=(p \circ f) \triangle g$.

Proof of the claim. Since $h_{0} \mid K$ is $(m, \omega)$-discrete, every $t \in h_{0}(K)$ has an open neighborhood $V_{t}$ in $M \times \mathbb{I}^{k}$ such that $h_{0}^{-1}\left(V_{t}\right) \cap K$ is $(m, \omega)$-discrete in $K$. Then $\nu=\left\{V_{t}: t \in h_{0}(K)\right\}$ forms an open cover of $h_{0}(K)$. Take $\gamma$ to be a locally finite open cover of $V=\bigcup \nu$ such that $\{\operatorname{St}(W, \gamma): W \in \gamma\}$ refines $\nu$. Let $h \mid K$ be a map $\gamma$-close to $h_{0} \mid K$, where $h=(p \circ f) \triangle g$ with $g \in C\left(X, \mathbb{I}^{k}\right)$. If $W \in \gamma$, then $h_{0}\left(h^{-1}(W) \cap K\right) \subset \operatorname{St}(W, \gamma)$. But $\operatorname{St}(W, \gamma)$ is contained in $V_{t}$ for some $t \in h_{0}(K)$. Consequently, $h^{-1}(W) \cap K \subset h_{0}^{-1}\left(V_{t}\right) \cap K$. The last inclusion implies that $h^{-1}(W) \cap K$ is $(m, \omega)$-discrete in $K$ because $h_{0}^{-1}\left(V_{t}\right) \cap K$ is. Therefore, $h \mid K$ is $(m, \omega)$-discrete. To finish the proof of the claim observe that $h \mid K$ being $(m, \omega)$-discrete implies $(f \triangle g) \mid K$ is also $(m, \omega)$-discrete, i.e. $g \in C_{(m, \omega)}\left(X \mid K, \mathbb{I}^{k}\right)$.

We continue with the proof of Lemma 2.5. Let $\varrho$ be the metric on $M \times \mathbb{I}^{k}$ defined by $\varrho\left(t_{1}, t_{2}\right)=d\left(z_{1}, z_{2}\right)+d_{k}\left(w_{1}, w_{2}\right)$, where $t_{i}=\left(z_{i}, w_{i}\right), i=1,2$. Let $\alpha_{1}: K \rightarrow(0, \infty)$ be the function $\alpha_{1}(x)=2^{-1} \sup \left\{\varrho\left(h_{0}(x), V \backslash W\right): W \in \gamma\right\}$. Since $h_{0}(K) \subset V$ and $\gamma$ is a locally finite open cover of $V, \alpha_{1}$ is continuous. Moreover, if $h=(p \circ f) \triangle g$ with $g \in C\left(X, \mathbb{I}^{k}\right)$ and $\varrho\left(h_{0}(x), h(x)\right) \leq \alpha_{1}(x)$ for every $x \in K$, then $h \mid K$ is $\gamma$-close to $h_{0} \mid K$. According to the claim, the last relation yields $g \in C_{(m, \omega)}\left(X \mid K, \mathbb{I}^{k}\right)$. We finally take a continuous extension $\alpha: X \rightarrow(0, \infty)$ of $\alpha_{1}$. Observe that $d_{k}\left(g_{0}(x), g(x)\right)=\varrho\left(h_{0}(x), h(x)\right)$ for every $x \in X$. Therefore, $\bar{B}\left(g_{0}, \alpha\right) \subset C_{(m, \omega)}\left(X \mid K, \mathbb{I}^{k}\right)$.

LEMMA 2.6. If $C\left(X, \mathbb{I}^{k}\right)$ is equipped with the uniform convergence topology, then the set-valued map $\psi_{(m, \omega)}: Y \rightarrow 2^{C\left(X, \mathbb{I}^{k}\right)}$, defined by the formula $\psi_{(m, \omega)}(y)=C\left(X, \mathbb{I}^{k}\right) \backslash C_{(m, \omega)}\left(X \mid f^{-1}(y), \mathbb{I}^{k}\right)$, has a closed graph.

Proof. Let $G=\bigcup\left\{y \times \psi_{(m, \omega)}(y): y \in Y\right\} \subset Y \times C\left(X, \mathbb{I}^{k}\right)$ be the graph of $\psi_{(m, \omega)}$ and $\left(y_{0}, g_{0}\right) \in\left(Y \times C\left(X, \mathbb{I}^{k}\right)\right) \backslash G$. We are going to show that $\left(y_{0}, g_{0}\right)$ has a neighborhood in $Y \times C\left(X, \mathbb{I}^{k}\right)$ which does not meet $G$. 
Since $\left(y_{0}, g_{0}\right) \notin G, g_{0} \notin \psi_{(m, \omega)}\left(y_{0}\right)$. Hence, $g_{0} \in C_{(m, \omega)}\left(X \mid f^{-1}\left(y_{0}\right), \mathbb{I}^{k}\right)$ and, by Lemma 2.3 , there exists a neighborhood $U$ of $y_{0}$ in $Y$ with $g_{0} \mid f^{-1}(U)$ being $(m, \omega)$-discrete, in particular, $g_{0} \in C_{(m, \omega)}\left(X \mid f^{-1}(U), \mathbb{I}^{k}\right)$. We can assume that $U \subset Y$ is closed, hence so is $f^{-1}(U)$ in $X$. Then, according to Lemma 2.5, $C_{(m, \omega)}\left(X \mid f^{-1}(U), \mathbb{I}^{k}\right)$ is open in $C\left(X, \mathbb{I}^{k}\right)$ with respect to the source limitation topology. Consequently, there exists a continuous positive function $\alpha$ on $X$ such that $\bar{B}\left(g_{0}, \alpha\right)$ is contained in $C_{(m, \omega)}\left(X \mid f^{-1}(U), \mathbb{I}^{k}\right)$. Since $f^{-1}\left(y_{0}\right)$ is compact, $2 \delta=\min \left\{\alpha(x): x \in f^{-1}\left(y_{0}\right)\right\}>0$ and $H=$ $\left\{x \in f^{-1}(U): \alpha(x)>\delta\right\}$ is a neighborhood of $f^{-1}\left(y_{0}\right)$. Therefore, there exists a closed neighborhood $V$ of $y_{0}$ in $Y$ with $f^{-1}(V) \subset H$ (we use again the fact that $f$ is a closed map). Let $B_{\delta}\left(g_{0}\right)$ be the open ball in $C\left(X, \mathbb{I}^{k}\right)$ (with respect to the uniform metric $D_{k}$ ) with center $g_{0}$ and radius $\delta$. Since $W=V \times B_{\delta}\left(g_{0}\right)$ is a neighborhood of $\left(y_{0}, g_{0}\right)$ in $Y \times C\left(X, \mathbb{I}^{k}\right)$, the following claim completes the proof.

Claim. $W \cap G=\emptyset$.

Suppose $(y, g) \in W \cap G$ for some $(y, g) \in Y \times C\left(X, \mathbb{I}^{k}\right)$. Then $y \in V$ and

$$
d_{k}\left(g(x), g_{0}(x)\right) \leq \delta<\alpha(x) \quad \text { for every } x \in f^{-1}(V) .
$$

Let us show that the existence of a function $g_{1} \in C\left(X, \mathbb{I}^{k}\right)$ such that

$$
g_{1} \in \bar{B}\left(g_{0}, \alpha\right) \quad \text { and } \quad g_{1}\left|f^{-1}(V)=g\right| f^{-1}(V)
$$

contradicts the assumption $(y, g) \in W \cap G$. Indeed, $g_{1} \in \bar{B}\left(g_{0}, \alpha\right)$ yields $g_{1} \in C_{(m, \omega)}\left(X \mid f^{-1}(U), \mathbb{I}^{k}\right)$ and, since $f^{-1}(y) \subset f^{-1}(U)$, we have $g_{1} \in$ $C_{(m, \omega)}\left(X \mid f^{-1}(y), \mathbb{I}^{k}\right)$. So, $g \in C_{(m, \omega)}\left(X \mid f^{-1}(y), \mathbb{I}^{k}\right)$ because $g_{1} \mid f^{-1}(y)=$ $g \mid f^{-1}(y)$ (recall that $\left.f^{-1}(y) \subset f^{-1}(V)\right)$. On the other hand, $(y, g) \in G$ implies $g \in \psi_{(m, \omega)}(y)$, i.e. $g \notin C_{(m, \omega)}\left(X \mid f^{-1}(y), \mathbb{I}^{k}\right)$.

Therefore, the proof is reduced to finding $g_{1}$ satisfying (2). And this can be done by using the convex-valued selection theorem of Michael [21]. Define the set-valued map $\Phi: X \rightarrow \mathcal{F}_{\mathrm{c}}\left(\mathbb{I}^{k}\right)$ by $\Phi(x)=g(x)$ if $x \in f^{-1}(V)$ and $\Phi(x)=\bar{B}_{\alpha(x)}\left(g_{0}(x)\right)$ otherwise. Here, $\mathcal{F}_{\mathrm{c}}\left(\mathbb{I}^{k}\right)$ denotes the convex closed subsets of $\mathbb{I}^{k}$ and $\bar{B}_{\alpha(x)}\left(g_{0}(x)\right)$ is the closed ball in $\mathbb{I}^{k}$ with center $g_{0}(x)$ and radius $\alpha(x)$. By $(1), g(x) \in \bar{B}_{\alpha(x)}\left(g_{0}(x)\right)$ for all $x \in f^{-1}(V)$. The last condition, together with the definition of $\Phi$ outside $f^{-1}(V)$, implies that $\Phi$ is lower semicontinuous (i.e., $\{x \in X: \Phi(x) \cap O \neq \emptyset\}$ is open in $X$ for any open set $\left.O \subset \mathbb{I}^{k}\right)$. Then, by the above mentioned Michael theorem, $\Phi$ admits a continuous selection $g_{1}$. Since $g_{1}(x) \in \Phi(x)$ for any $x \in X$, we have $g_{1}\left|f^{-1}(V)=g\right| f^{-1}(V)$ and $g_{1} \in \bar{B}\left(g_{0}, \alpha\right)$.

LEMMA 2.7. Let $K$ and $M$ be compact spaces such that $\operatorname{dim} K \leq n$ and $M$ is metrizable. Then for every $\gamma \in \operatorname{cov}(K)$ and $0 \leq k \leq n$ the set of all maps $h \in C\left(M \times K, \mathbb{I}^{k}\right)$ with each $h \mid(\{z\} \times K), z \in M$, being $(n-k, \gamma)$ - 
discrete (as a map from $K$ into $\left.\mathbb{I}^{k}\right)$ is dense in $C\left(M \times K, \mathbb{I}^{k}\right)$ with respect to the uniform convergence topology.

Proof. Suppose first that $K$ is metrizable and let $p_{M}: M \times K \rightarrow M$ and $p_{K}: M \times K \rightarrow K$ be the projections. Then, by Hurewicz's theorem [18], there exists a 0-dimensional map $h^{*}: K \rightarrow \mathbb{I}^{n}$. Consequently, $g^{*}=h^{*} \circ p_{K}$ is a map from $M \times K$ into $\mathbb{I}^{n}$ such that $p_{M} \triangle g^{*}: M \times K \rightarrow M \times \mathbb{I}^{n}$ is also 0-dimensional. According to Levin's [19] and Sternfeld's [29] results, the existence of such a map $g^{*}$ implies that the set $\mathcal{M}_{n}$ of all maps $g \in$ $C\left(M \times K, \mathbb{I}^{n}\right)$ with $\operatorname{dim}\left(p_{M} \triangle g\right) \leq 0$ is dense in $C\left(M \times K, \mathbb{I}^{n}\right)$ with respect to the uniform convergence topology. If $q: \mathbb{I}^{n} \rightarrow \mathbb{I}^{k}$ is the projection generated by the first $k$ coordinates, then the map $g \mapsto q \circ g$ is a continuous surjection from $C\left(M \times K, \mathbb{I}^{n}\right)$ onto $C\left(M \times K, \mathbb{I}^{k}\right)$ (both equipped with the uniform convergence topology), so $\mathcal{M}_{k}=\left\{q \circ g: g \in \mathcal{M}_{n}\right\}$ is dense in $C\left(M \times K, \mathbb{I}^{k}\right)$. Moreover, since $\operatorname{dim} q=n-k$ and each $p_{M} \triangle g, g \in \mathcal{M}_{n}$, is 0-dimensional, $\operatorname{dim}\left(p_{M} \triangle h\right) \leq n-k$ for any $h \in \mathcal{M}_{k}$ (the last conclusion follows from the Hurewicz theorem on closed maps which lower dimension [16]). Therefore, $h_{z}=h \mid(\{z\} \times K)$ is an $(n-k)$-dimensional map for every $z \in M$ and $h \in \mathcal{M}_{k}$.

Let us show that any such $h_{z}$ is $(n-k, \gamma)$-discrete. Indeed, for fixed $y \in h_{z}(K)$ we have $\operatorname{dim} h_{z}^{-1}(y) \leq n-k$. So, there exists $\nu \in \operatorname{cov}\left(h_{z}^{-1}(y)\right)$ refining $\gamma$ such that $\operatorname{ord}(\nu) \leq n-k+1$. Applying Lemma 2.1, we obtain an $(n-k, \gamma)$-discrete set $W_{y}$ in $K$ which contains $h_{z}^{-1}(y)$. Finally, choose a neighborhood $V_{y}$ of $y$ in $\mathbb{I}^{k}$ such that $h_{z}^{-1}\left(V_{y}\right) \subset W_{y}$ and observe that $h_{z}^{-1}\left(V_{y}\right)$ is $(n-k, \gamma)$-discrete.

Suppose now $K$ is not metrizable and fix $\delta>0$ and $h_{0} \in C\left(M \times K, \mathbb{I}^{k}\right)$. We are going to find $h \in C\left(M \times K, \mathbb{I}^{k}\right)$ satisfying the requirement of the lemma and such that $h$ is $\delta$-close to $h_{0}$. To this end, represent $K$ as the limit space of a $\sigma$-complete inverse system $\mathcal{S}=\left\{K_{\beta}, \pi_{\beta}^{\beta+1}: \beta \in B\right\}$ such that each $K_{\beta}$ is a metrizable compactum with $\operatorname{dim} K_{\beta} \leq n$. Applying standard inverse spectra arguments (see [4]), we can find $\theta \in B, \gamma_{1} \in \operatorname{cov}\left(K_{\theta}\right)$ and $h_{\theta} \in C\left(M \times K_{\theta}, \mathbb{I}^{k}\right)$ such that $h_{\theta} \circ\left(\operatorname{id}_{M} \times \pi_{\theta}\right)=h_{0}$ and $\pi_{\theta}^{-1}\left(\gamma_{1}\right)$ refines $\gamma$, where $\pi_{\theta}: K \rightarrow K_{\theta}$ denotes the $\theta$ th limit projection. Then, by the previous case, there exists a map $h_{1} \in C\left(M \times K_{\theta}, \mathbb{I}^{k}\right)$ which is $\delta$-close to $h_{\theta}$ and $h_{1} \mid\left(\{z\} \times K_{\theta}\right)$ is $\left(n-k, \gamma_{1}\right)$-discrete. It follows from our construction that $h=h_{1} \circ\left(\operatorname{id}_{M} \times \pi_{\theta}\right)$ is $\delta$-close to $h_{0}$ and $h \mid(\{z\} \times K)$ is $(n-k, \gamma)$ discrete.

Recall that a closed subset $F$ of the metrizable space $M$ is said to be a $Z$-set in $M$ if the set $C(Q, M \backslash F)$ is dense in $C(Q, M)$ with respect to the uniform convergence topology, where $Q$ denotes the Hilbert cube. If, in the above definition, $Q$ is replaced by $\mathbb{I}^{m}, m \in \mathbb{N} \cup\{0\}$, we say that $F$ is a $Z_{m}$-set in $M$. 
LEMMA 2.8. Let $\alpha: X \rightarrow(0, \infty)$ be a positive continuous function and $g_{0} \in C\left(X, \mathbb{I}^{k}\right)$. Then $\psi_{(n-k, \omega)}(y) \cap \bar{B}\left(g_{0}, \alpha\right)$ is a $Z$-set in $\bar{B}\left(g_{0}, \alpha\right)$ for every $y \in Y$, where $\bar{B}\left(g_{0}, \alpha\right)$ is considered as a subspace of $C\left(X, \mathbb{I}^{k}\right)$ with the uniform convergence topology.

Proof. In this proof all function spaces are equipped with the uniform convergence topology. Since, by Lemma $2.6, \psi_{(n-k, \omega)}$ has a closed graph, each $\psi_{(n-k, \omega)}(y)$ is closed in $C\left(X, \mathbb{I}^{k}\right)$. Hence, $\psi_{(n-k, \omega)}(y) \cap \bar{B}\left(g_{0}, \alpha\right)$ is closed in $\bar{B}\left(g_{0}, \alpha\right)$. We need to show that, for fixed $y \in Y, \delta>0$ and a map $u: Q \rightarrow \bar{B}\left(g_{0}, \alpha\right)$ there exists a map $v: Q \rightarrow \bar{B}\left(g_{0}, \alpha\right) \backslash \psi_{(n-k, \omega)}(y)$ which is $\delta$-close to $u$ with respect to the uniform metric $D_{k}$.

To this end, observe first that $u$ generates $h \in C\left(Q \times X, \mathbb{I}^{k}\right), h(z, x)=$ $u(z)(x)$, such that $d_{k}\left(h(z, x), g_{0}(x)\right) \leq \alpha(x)$ for any $(z, x) \in Q \times X$. Since $f^{-1}(y)$ is compact, we can find $\lambda \in(0,1)$ such that $\lambda \sup \{\alpha(x)$ : $\left.x \in f^{-1}(y)\right\}<\delta / 2$. Now, define $h_{1} \in C\left(Q \times f^{-1}(y), \mathbb{I}^{k}\right)$ by $h_{1}(z, x)=$ $(1-\lambda) h(z, x)+\lambda g_{0}(x)$. Then, for every $(z, x) \in Q \times f^{-1}(y)$, we have

$$
d_{k}\left(h_{1}(z, x), g_{0}(x)\right) \leq(1-\lambda) \alpha(x)<\alpha(x)
$$

and

$$
d_{k}\left(h_{1}(z, x), h(z, x)\right) \leq \lambda \alpha(x)<\delta / 2 .
$$

Let $q<\min \{r, \delta / 2\}$, where $r$ is the positive number $\inf \left\{\alpha(x)-d_{k}\left(h_{1}(z, x)\right.\right.$, $\left.\left.g_{0}(x)\right):(z, x) \in Q \times f^{-1}(y)\right\}$. Since $\operatorname{dim} f^{-1}(y) \leq n$, by Lemma 2.7 (applied to the product $\left.Q \times f^{-1}(y)\right)$, there is a map $h_{2} \in C\left(Q \times f^{-1}(y)\right.$, $\left.\mathbb{I}^{k}\right)$ such that $d_{k}\left(h_{2}(z, x), h_{1}(z, x)\right)<q$ and $h_{2} \mid\left(\{z\} \times f^{-1}(y)\right)$ is an $(n-k, \omega)$-discrete map for each $(z, x) \in Q \times f^{-1}(y)$. Then, by (3) and (4), for all $(z, x) \in Q \times f^{-1}(y)$ we have

$$
d_{k}\left(h_{2}(z, x), h(z, x)\right)<\delta \quad \text { and } \quad d_{k}\left(h_{2}(z, x), g_{0}(x)\right)<\alpha(x) .
$$

Because both $Q$ and $f^{-1}(y)$ are compact, $u_{2}(z)(x)=h_{2}(z, x)$ defines the map $u_{2}: Q \rightarrow C\left(f^{-1}(y), \mathbb{I}^{k}\right)$. The required map $v$ will be obtained as a lifting of $u_{2}$. The restriction map $\pi: \bar{B}\left(g_{0}, \alpha\right) \rightarrow C\left(f^{-1}(y), \mathbb{I}^{k}\right), \pi(g)=g \mid f^{-1}(y)$, is obviously continuous (with respect to the uniform convergence topology).

Claim. $\pi: \bar{B}\left(g_{0}, \alpha\right) \rightarrow \pi\left(\bar{B}\left(g_{0}, \alpha\right)\right)$ is an open map.

It is enough to show that

$$
\pi\left(\bar{B}\left(g_{0}, \alpha\right) \cap B_{\varepsilon}(g)\right)=\pi\left(\bar{B}\left(g_{0}, \alpha\right)\right) \cap B_{\varepsilon}(\pi(g))
$$

for every $g \in \bar{B}\left(g_{0}, \alpha\right)$ and $\varepsilon>0$, where $B_{\varepsilon}(g)$ and $B_{\varepsilon}(\pi(g))$ are open balls, respectively, in $C\left(X, \mathbb{I}^{k}\right)$ and $C\left(f^{-1}(y), \mathbb{I}^{k}\right)$, both with the uniform metric generated by $d_{k}$. Let $p \in \pi\left(\bar{B}\left(g_{0}, \alpha\right)\right) \cap B_{\varepsilon}(\pi(g))$. Then $d_{k}\left(p(x), g_{0}(x)\right) \leq \alpha(x)$ and $d_{k}(p(x), g(x))<\eta<\varepsilon$ for every $x \in f^{-1}(y)$ and some positive number $\eta$. Define the closed-and-convex-valued map $\Phi: X \rightarrow \mathcal{F}_{\mathrm{c}}\left(\mathbb{I}^{k}\right)$ by $\Phi(x)=p(x)$ if $x \in f^{-1}(y)$ and $\Phi(x)=\overline{B_{\alpha(x)}\left(g_{0}(x)\right) \cap B_{\eta}(g(x))}$ if $x \notin f^{-1}(y)$ (recall 
that $B_{\alpha(x)}\left(g_{0}(x)\right)$ and $B_{\eta(x)}(g(x))$ are open balls in $\left.\mathbb{I}^{k}\right)$. Since $g \in \bar{B}\left(g_{0}, \alpha\right)$, $\Phi(x) \neq \emptyset$ for every $x \in X$. Moreover, since $\alpha, g$ and $g_{0}$ are continuous, $\Phi$ is lower semicontinuous. Therefore, by Michael's convex-valued selection theorem, $\Phi$ admits a selection $g_{1} \in C\left(X, \mathbb{I}^{k}\right)$. Then $\pi\left(g_{1}\right)=p$ and $g_{1} \in$ $\bar{B}\left(g_{0}, \alpha\right) \cap B_{\varepsilon}(g)$. So, $\pi\left(\bar{B}\left(g_{0}, \alpha\right)\right) \cap B_{\varepsilon}(\pi(g)) \subset \pi\left(\bar{B}\left(g_{0}, \alpha\right) \cap B_{\varepsilon}(g)\right)$ and, because the converse inclusion is trivial, we are done.

Before completing the final step of our proof, note that $u_{2}(z) \in$ $\pi\left(\bar{B}\left(g_{0}, \alpha\right)\right)$ for every $z \in Q$ (indeed, consider the lower semicontinuous set-valued map $\phi: X \rightarrow \mathcal{F}_{\mathrm{c}}\left(\mathbb{I}^{k}\right), \phi(x)=u_{2}(z)(x)$ for $x \in f^{-1}(y)$ and $\phi(x)=\overline{B_{\alpha(x)}\left(g_{0}(x)\right)}$ for $x \notin f^{-1}(y)$, and take any continuous selection $g$ of $\phi)$. Now, we are going to lift the map $u_{2}$ to a map $v: Q \rightarrow \bar{B}\left(g_{0}, \alpha\right)$ such that $v$ is $\delta$-close to $u$.

To this end, define $\theta: Q \rightarrow \mathcal{F}_{\mathrm{c}}\left(C\left(X, \mathbb{I}^{k}\right)\right)$ by $\theta(z)=\overline{\pi^{-1}\left(u_{2}(z)\right) \cap B_{\delta}(u(z))}$. The first inequality in (5) implies that $u_{2}(z) \in B_{\delta}(\pi(u(z)))$ for every $z \in Q$. Since each $u_{2}(z)$ belongs to $\pi\left(\bar{B}\left(g_{0}, \alpha\right)\right)$, by $(6), \theta(z) \neq \emptyset, z \in Q$. On the other hand, since $\pi$ is open, by [21, Example 1.1* and Proposition 2.5], $\theta$ is lower semicontinuous. Obviously, every image $\theta(z)$ is convex and closed in $C\left(X, \mathbb{I}^{k}\right)$, which is, in its turn, closed and convex in the Banach space of all bounded continuous functions from $X$ into $\mathbb{R}^{k}$. Therefore, using again the Michael selection theorem [21, Theorem 3.2" $]$, we can find a continuous selection $v: Q \rightarrow C\left(X, \mathbb{I}^{k}\right)$ for $\theta$. Then $v$ maps $Q$ into $\bar{B}\left(g_{0}, \alpha\right)$ and $v$ is $\delta$-close to $u$. Moreover, for any $z \in Q$ we have $\pi(v(z))=u_{2}(z)$, and $u_{2}(z)$, being the restriction $h_{2} \mid\left(\{z\} \times f^{-1}(y)\right)$, is $(n-k, \omega)$-discrete. Hence, $v(z) \in C_{(n-k, \omega)}\left(X \mid f^{-1}(y), \mathbb{I}^{k}\right), z \in Q$, i.e. $v(Q) \subset \bar{B}\left(g_{0}, \alpha\right) \backslash \psi_{(n-k, \omega)}(y)$.

Lemma 2.9. If $Y$ is a $C$-space, then $C_{(n-k, \omega)}\left(X, \mathbb{I}^{k}\right)$ is dense in $C\left(X, \mathbb{I}^{k}\right)$ with respect to the source limitation topology.

Proof. It suffices to show that, for fixed $g_{0} \in C\left(X, \mathbb{I}^{k}\right)$ and a positive continuous function $\alpha: X \rightarrow(0, \infty)$, there exists $g \in \bar{B}\left(g_{0}, \alpha\right) \cap C_{(n-k, \omega)}\left(X, \mathbb{I}^{k}\right)$. We equip $C\left(X, \mathbb{I}^{k}\right)$ with the uniform convergence topology and consider the constant (and hence, lower semicontinuous) map $\phi: Y \rightarrow \mathcal{F}_{\mathrm{c}}\left(C\left(X, \mathbb{I}^{k}\right)\right.$ ), $\phi(y)=\bar{B}\left(g_{0}, \alpha\right)$. According to Lemma $2.8, \bar{B}\left(g_{0}, \alpha\right) \cap \psi_{(n-k, \omega)}(y)$ is a $Z$ set in $\bar{B}\left(g_{0}, \alpha\right)$ for every $y \in Y$. So, we have a lower semicontinuous map $\phi: Y \rightarrow \mathcal{F}_{\mathrm{c}}(E)$ and a map $\psi_{(n-k, \omega)}: Y \rightarrow 2^{E}$ such that $\psi_{(n-k, \omega)}$ has a closed graph (see Lemma 2.6) and $\phi(y) \cap \psi_{(n-k, \omega)}(y)$ is a $Z$-set in $\phi(y)$ for each $y \in Y$, where $E$ is the Banach space of all bounded continuous maps from $Y$ into $\mathbb{R}^{k}$. Therefore, we can apply [14, Theorem 1.1] to obtain a continuous map $h: Y \rightarrow E$ with $h(y) \in \phi(y) \backslash \psi_{(n-k, \omega)}(y)$ for every $y \in Y$ (Theorem 1.1 of [14] was proved under the assumption that $\psi_{(n-k, \omega)}$ has nonempty values, but the proof works without this restriction). Observe that $h$ is a map from $Y$ into $\bar{B}\left(g_{0}, \alpha\right)$ such that $h(y) \notin \psi_{(n-k, \omega)}(y)$ for every $y \in Y$, i.e. 
$h(y) \in \bar{B}\left(g_{0}, \alpha\right) \cap C_{(n-k, \omega)}\left(X \mid f^{-1}(y), \mathbb{I}^{k}\right), y \in Y$. Then $g(x)=h(f(x))(x)$, $x \in X$, defines a map $g \in \bar{B}\left(g_{0}, \alpha\right)$ such that $g \in C_{(n-k, \omega)}\left(X \mid f^{-1}(y), \mathbb{I}^{k}\right)$ for every $y \in Y$. Hence, by Corollary $2.4, g \in C_{(n-k, \omega)}\left(X, \mathbb{I}^{k}\right)$.

3. Proof of Theorem 1.3. The following proposition proves Theorem 1.3 in the special case when $f$ is $\sigma$-perfect.

Proposition 3.1. Let $f: X \rightarrow Y$ be a $\sigma$-perfect map of metrizable spaces with $\operatorname{dim} f \leq n$ and $Y$ being a $C$-space. Then the set of all maps $g: X \rightarrow \mathbb{I}^{n}$ such that $\operatorname{dim}(f \triangle g)=0$ is dense and $G_{\delta}$ in $C\left(X, \mathbb{I}^{n}\right)$ with respect to the source limitation topology.

Proof. All function spaces in this proof are considered with the source limitation topology. Let $X$ be the union of closed sets $X_{i}, i=1,2, \ldots$, such that each restriction $f_{i}=f \mid X_{i}$ is perfect and $Y_{i}=f\left(X_{i}\right)$ is closed in $Y$. Fix a sequence $\left\{\omega_{q}\right\}$ of open covers of $X$ with $\operatorname{mesh}\left(\omega_{q}\right)<q^{-1}$. Every $Y_{i}$ is a $C$-space (being a closed set in $Y$ ), so we can apply Lemma 2.9 to the maps $f_{i}: X_{i} \rightarrow Y_{i}$ and conclude that $H_{i}=\bigcap_{q=1}^{\infty} C_{\left(0, \omega_{q}\right)}\left(X_{i}, \mathbb{I}^{n}\right)$ is dense and $G_{\delta}$ in $C\left(X_{i}, \mathbb{I}^{n}\right), i \in \mathbb{N}$. Here, $C_{\left(0, \omega_{q}\right)}\left(X_{i}, \mathbb{I}^{n}\right)$ consists of all $h \in C\left(X_{i}, \mathbb{I}^{n}\right)$ such that $f_{i} \triangle h$ is $\left(0, \omega_{q}\right)$-discrete. Since all restriction maps $p_{i}: C\left(X, \mathbb{I}^{n}\right) \rightarrow C\left(X_{i}, \mathbb{I}^{n}\right)$, $p_{i}(g)=g \mid X_{i}$, are continuous, open and surjective, the sets $C_{i}=p_{i}^{-1}\left(H_{i}\right)$ are dense and $G_{\delta}$ in $C\left(X, \mathbb{I}^{n}\right)$, and hence so is the intersection $\bigcap_{i=1}^{\infty} C_{i}$. It remains to observe that $g \in \bigcap_{i=1}^{\infty} C_{i}$ if and only if $\operatorname{dim}\left(f_{i} \triangle g_{i}\right)=0$ for every $i$, where $g_{i}=g \mid X_{i}$. Hence, by the countable sum theorem, $g \in \bigcap_{i=1}^{\infty} C_{i}$ if and only if $\operatorname{dim}(f \triangle g)=0$.

We now continue with the proof of the first part of Theorem 1.3. Suppose $f: X \rightarrow Y$ is a closed $n$-dimensional surjection with both $X$ and $Y$ metrizable and $Y$ a $C$-space. By the Vainstein lemma [12], the boundary $\operatorname{Fr} f^{-1}(y)$ of every $f^{-1}(y)$ is compact. Defining $F(y)$ to be $\operatorname{Fr} f^{-1}(y)$ if $\operatorname{Fr} f^{-1}(y) \neq \emptyset$, and an arbitrary point from $f^{-1}(y)$ otherwise, we obtain the set $X_{0}=\bigcup\{F(y): y \in Y\}$ such that $X_{0} \subset X$ is closed and the restriction $f \mid X_{0}: X_{0} \rightarrow Y$ is a perfect surjection. Moreover, each $f^{-1}(y) \backslash X_{0}$ is open in $X$, so $\operatorname{dim}\left(X \backslash X_{0}\right) \leq n$.

Represent $X \backslash X_{0}$ as the union of countably many closed sets $X_{i} \subset X$ and for each $i=0,1,2, \ldots$ let $p_{i}: C\left(X, \mathbb{I}^{n}\right) \rightarrow C\left(X_{i}, \mathbb{I}^{n}\right)$ be the restriction map. By Proposition 3.1, the set $C_{0}$ consisting of all $g \in C\left(X, \mathbb{I}^{n}\right)$ with $(f \triangle g) \mid X_{0}$ being 0-dimensional is dense and $G_{\delta}$ in $C\left(X, \mathbb{I}^{n}\right)$ with respect to the source limitation topology. Consequently, $C_{0}$ is uniformly dense in $C\left(X, \mathbb{I}^{n}\right)$. On the other hand, since $\operatorname{dim} X_{i} \leq n$ for every $i=1,2, \ldots$, the set $H_{i} \subset C\left(X_{i}, \mathbb{I}^{n}\right)$ of all uniformly 0-dimensional maps is dense and $G_{\delta}$ in $C\left(X_{i}, \mathbb{I}^{n}\right)$ with respect to the uniform convergence topology [17] (recall that a map $h: X_{i} \rightarrow \mathbb{I}^{n}$ is uniformly 0 -dimensional if for every $\varepsilon>0$ there exists 
$\eta>0$ such that if $T \subset \mathbb{I}^{n}$ and $\operatorname{diam}(T) \leq \eta$, then $h^{-1}(T)$ is covered by a disjoint open family in $X_{i}$ consisting of sets with diameter $\left.\leq \varepsilon\right)$. Because $p_{i}$ are open and continuous surjections when $C\left(X, \mathbb{I}^{n}\right)$ and $C\left(X_{i}, \mathbb{I}^{n}\right)$ carry the uniform convergence topology, all $C_{i}=p_{i}^{-1}\left(H_{i}\right), i=1,2, \ldots$, are uniformly dense and $G_{\delta}$ in $C\left(X, \mathbb{I}^{n}\right)$. Therefore, $C_{\infty}=\bigcap_{i=0}^{\infty} C_{i}$ is $G_{\delta}$ in $C\left(X, \mathbb{I}^{n}\right)$ with respect to the source limitation topology. Moreover, $f \triangle g$ is 0 -dimensional for every $g \in C_{\infty}$.

It remains to show that $C_{\infty}$ is uniformly dense in $C\left(X, \mathbb{I}^{n}\right)$. For every $g \in C_{0}$ let $H(g)=\left\{h \in C\left(X, \mathbb{I}^{n}\right): h\left|X_{0}=g\right| X_{0}\right\}$. Obviously, $C_{0}=\bigcup\{H(g):$ $\left.g \in C_{0}\right\}$ and each $H(g)$ is uniformly closed in $C\left(X, \mathbb{I}^{n}\right)$. So, $C_{\infty}$ is the union of the sets $A(g)=\bigcap_{i=1}^{\infty} C_{i} \cap H(g), g \in C_{0}$. For fixed $g \in C_{0}$ and $i=1,2, \ldots$, let $p_{i}(g)=p_{i} \mid H(g)$. Using the fact that $X_{0}$ and $X_{i}$ are closed disjoint subsets of $X$, one can show that every $p_{i}(g): H(g) \rightarrow C\left(X_{i}, \mathbb{I}^{n}\right)$ is a uniformly continuous open surjection. Hence, $H(g) \cap C_{i}$ is dense and $G_{\delta}$ in $H(g)$ with respect to the uniform convergence topology, being the preimage of $H_{i}$ under $p_{i}(g)$. Therefore, $A(g)$ is uniformly dense in $H(g)$ (recall that $H(g)$ is uniformly closed in $C\left(X, \mathbb{I}^{n}\right)$, so it has the Baire property). We finally observe that the uniform density of $C_{0}$ in $C\left(X, \mathbb{I}^{n}\right)$ and the uniform density of $A(g)$ in $H(g)$ for each $g \in C_{0}$ yield the uniform density of $C_{\infty}$ in $C\left(X, \mathbb{I}^{n}\right)$.

4. Proof of Theorem 1.4. It suffices to prove this theorem for closed maps, so we suppose that $f: X \rightarrow Y$ is a closed surjection. If $A_{n-1}$ is constructed, then for $k<n-1$, we can find an $F_{\sigma}$-subset $A_{k} \subset A_{n-1}$ with $\operatorname{dim} A_{k} \leq k$ and $\operatorname{dim}\left(A_{n-1} \backslash A_{k}\right) \leq n-k-2$ (this can be accomplished by induction, the first step is to represent $A_{n-1}$ as the union of 0-dimensional $G_{\delta}$-subsets $B_{j}, j=1, \ldots, n$ and to set $A_{n-2}=\bigcup_{j=1}^{n-1} B_{j}$ ). Therefore, we only need to construct $A_{n-1}$. To this end, we first establish the following analogue of Sternfeld's [29, Lemma 1] which was proved for compact metrizable spaces.

Lemma 4.1. Let $M$ be metrizable and $K$ a compact metric space with $\operatorname{dim} K \leq n$. Then there exists a $F_{\sigma}$-subset $B \subset M \times K$ such that $\operatorname{dim} B \leq$ $n-1$ and $\pi_{M} \mid(M \times K) \backslash B$ is 0-dimensional, where $\pi_{M}: M \times K \rightarrow M$ is the projection.

Proof. As in [29], the proof can be reduced to the case $n=1$ and $K=$ $[0,1]$. So, we are going to show the existence of a 0 -dimensional $F_{\sigma}$-subset $B$ of $M \times \mathbb{I}$ such that each set $(\{y\} \times \mathbb{I}) \backslash B, y \in M$, is 0-dimensional, and that will complete the proof.

Let $h: Z \rightarrow M$ be a perfect surjection with $Z$ being a 0 -dimensional metrizable space. Then, by [24, Proposition 9.1], there exists a map $g: Z \rightarrow$ $Q$ such that $h \triangle g: Z \rightarrow M \times Q$ is a closed embedding. Next, let $D$ be the Cantor set and take a surjection $p: D \rightarrow Q$ admitting an averaging 
operator between the function spaces $C(D)$ and $C(Q)$ [26] (such maps are called Milyutin maps). According to [6], there exists a lower semicontinuous compact-valued map $\phi: Q \rightarrow 2^{D}$ with $\phi(y) \subset p^{-1}(y)$ for every $y \in Q$. We can apply Michael's 0-dimensional selection theorem [22] to obtain a continuous selection $q$ for the map $\phi \circ g$. Obviously $h \triangle q: Z \rightarrow M \times D$ is a closed embedding, so $Z_{0}=(h \triangle q)(Z)$ is a 0-dimensional closed subset of $M \times D$. Finally, considering $D$ as a subset of $\mathbb{I}$, let $Z_{r}=\{(h(z), q(z)+r)$ : $z \in Z\} \subset M \times \mathbb{I}$ for every rational $r \in \mathbb{I}$, where addition $q(z)+r$ is taken in $\mathbb{R} \bmod 1$. Then each $Z_{r}$ is a closed subset of $M \times \mathbb{I}$ homeomorphic to $Z$, so $B=\bigcup\left\{Z_{r}: r\right.$ is rational $\}$ is 0 -dimensional and $F_{\sigma}$ in $M \times \mathbb{I}$. Moreover, $(\{y\} \times \mathbb{I}) \backslash B$ is also 0-dimensional for every $y \in M$.

Let us continue the proof of Theorem 1.4. As in the proof of Theorem 1.3, there are closed subsets $X_{i} \subset X, i=0,1,2, \ldots$, such that $f \mid X_{0}$ is a perfect map onto $Y$, each $X_{i}, i \geq 1$, is at most $n$-dimensional and $X \backslash X_{0}=\bigcup_{i=1}^{\infty} X_{i}$. For every $i \geq 1$ we choose an $(n-1)$-dimensional $F_{\sigma}$-set $H_{i} \subset X_{i}$ with $\operatorname{dim}\left(X_{i} \backslash H_{i}\right) \leq 0$.

A similar subset of $X_{0}$ can also be found. Indeed, let $f_{0}=f \mid X_{0}$ and take $g: X_{0} \rightarrow \mathbb{I}^{n}$ such that $f_{0} \triangle g: X_{0} \rightarrow Y \times \mathbb{I}^{n}$ is 0-dimensional (see Theorem 1.3). By Lemma 4.1, there exists an $F_{\sigma}$-set $B \subset Y \times \mathbb{I}^{n}$ with $\operatorname{dim} B \leq n-1$ and each $\left(\{y\} \times \mathbb{I}^{n}\right) \backslash B, y \in Y$, being 0 -dimensional. Then $H_{0}=$ $\left(f_{0} \triangle g\right)^{-1}(B)$ is $F_{\sigma}$ in $X_{0}$. Since $f_{0} \triangle g$ is perfect, by the generalized Hurewicz theorem on closed maps lowering dimension [28], we have $\operatorname{dim} H_{0} \leq n-1$ and $\operatorname{dim}\left(f_{0}^{-1}(y) \backslash H_{0}\right) \leq 0$ for every $y \in Y$.

Finally, set $A_{n-1}=\bigcup_{i=0}^{\infty} H_{i}$. Obviously, $\operatorname{dim} A_{n-1} \leq n-1$. On the other hand, each $f^{-1}(y) \backslash A_{n-1}, y \in Y$, is the union of its closed sets $F_{i}(y)=$ $f^{-1}(y) \cap X_{i} \backslash A_{n-1}, i \geq 0$. But $F_{0}(y)=f_{0}^{-1}(y) \backslash H_{0}$ and $F_{i}(y) \subset f^{-1}(y) \cap$ $\left(X_{i} \backslash H_{i}\right)$ for $i \geq 1$, so all $F_{i}(y)$ are 0 -dimensional. Consequently, $\operatorname{dim}\left(f^{-1}(y) \backslash\right.$ $\left.A_{n-1}\right) \leq 0$ for every $y \in Y$, i.e. $f \mid\left(X \backslash A_{n-1}\right)$ is 0 -dimensional.

5. Some applications. Our first application deals with extensional dimension introduced by Dranishnikov [7] (see also [3] and [8]). Let $K$ be a $C W$-complex and $X$ a normal space. We say that the extensional dimension of $X$ does not exceed $K$, written e-dim $X \leq K$, if every map $h: A \rightarrow K$, where $A \subset X$ is closed, can be extended to a map from $X$ into $K$ provided there exist a neighborhood $U$ of $A$ in $X$ and a map $g: U \rightarrow K$ extending $h$. Obviously, if $K$ is an absolute neighborhood extensor for $X$, then e- $\operatorname{dim} X \leq$ $K$ iff $K$ is an absolute extensor for $X$. In this notation, $\operatorname{dim} X \leq n$ is equivalent to e-dim $X \leq \mathbb{S}^{n}$. We also write e-dim $X \leq$ e-dim $Y$ if e-dim $Y \leq K$ implies e-dim $X \leq K$ for any $C W$-complex $K$.

Dranishnikov and Uspenskij [10] provided a generalization of the Hurewicz theorem on dimension lowering maps: if $f: X \rightarrow Y$ is an $n$-dimensio- 
nal surjection between compact finite-dimensional spaces, then e-dim $X \leq$ e- $\operatorname{dim}\left(Y \times \mathbb{I}^{n}\right)$; moreover, this statement holds for any compact spaces (not necessarily finite-dimensional) when $n=0$. We can improve this result as follows (see also [5] and [9] for other extension-dimensional variants of Hurewicz's theorem):

THEOREM 5.1. If $f: X \rightarrow Y$ is a perfect $n$-dimensional surjection such that $Y$ is a paracompact $C$-space, then $\mathrm{e}-\operatorname{dim} X \leq \mathrm{e}-\operatorname{dim}\left(Y \times \mathbb{I}^{n}\right)$.

Theorem 5.1 follows from Theorem 2.2 and the next proposition which can be extracted from the Dranishnikov and Uspenskij proof of their $[10$, Lemma 2.1 and Theorem 1.4].

Proposition 5.2. Let $K$ be a $C W$-complex and $X$ paracompact. If for any $\omega \in \operatorname{cov}(X)$ there exist a paracompact space $Z_{\omega}$ with e-dim $Z_{\omega} \leq K$ and a perfect $(0, \omega)$-discrete map $g: X \rightarrow Z_{\omega}$, then e-dim $X \leq K$.

Corollary 5.3. Let $f: X \rightarrow Y$ be a $\sigma$-closed $n$-dimensional surjection between metrizable spaces with $Y$ being a $C$-space. Then e-dim $X \leq$ $\mathrm{e}-\operatorname{dim}\left(Y \times \mathbb{I}^{n}\right)$.

Proof. Let $K$ be a $C W$-complex with e-dim $\left(Y \times \mathbb{I}^{n}\right) \leq K$. It suffices to show that e-dim $X \leq K$. Since extension dimension satisfies the countable sum theorem, the proof reduces to the case of $f$ closed. We can also assume that $K$ is an open subset of a normed space because every $C W$-complex is homotopy equivalent to such a set. Represent $X$ as the union of closed sets $X_{i} \subset X, i \geq 0$, such that $f \mid X_{0}$ is a perfect map onto $Y$ and $\operatorname{dim} X_{i} \leq n$ for each $i \geq 1$ (see the proof of Theorem 1.3). Then, by Theorem 5.1, e-dim $X_{0}$ $\leq K$. On the other hand, e-dim $\left(Y \times \mathbb{I}^{n}\right) \leq K$ implies that e-dim $\mathbb{I}^{n} \leq K$, in particular, every map from $\mathbb{S}^{n-1}$ into $K$ is extendable to a map from $\mathbb{I}^{n}$ into $K$. In other words, $K$ is $C^{n-1}$ and, as an open subset of a normed space, $K$ is also $L C^{n-1}$. It is well known that $L C^{n-1}$ and $C^{n-1}$ metrizable spaces are precisely the absolute extensors for $n$-dimensional metrizable spaces. Hence, e-dim $X_{i} \leq K$ for every $i \geq 1$. Finally, by the countable sum theorem for extensional dimension, we have e-dim $X \leq K$.

Another application is a parametric version of the Bogaty $\breve{1}$ decomposition theorem for $n$-dimensional metrizable spaces [2]: For every metrizable $n$ dimensional space $M$ there exist countably many 0-dimensional $G_{\delta}$-subsets $M_{k} \subset M$ such that $M=\bigcup_{i=1}^{n+1} M_{k(i)}$ for all pairwise distinct $k(1), \ldots, k(n+1)$ in $\mathbb{N}$.

Proposition 5.4. Let $f: X \rightarrow Y$ be a closed $n$-dimensional surjection between metrizable spaces with $Y$ a $C$-space. Then there exists a sequence $\left\{A_{k}\right\}$ of $G_{\delta}$-subsets of $X$ such that every restriction $f \mid A_{k}$ is 0 -dimensional and for any $P \subset \mathbb{N}$ of cardinality $n+1$ we have $X=\bigcup_{k \in P} A_{k}$. 
Proof. Take closed sets $X_{i} \subset X, i \geq 0$, and a map $g: X \rightarrow \mathbb{I}^{n}$ such that $f \mid X_{0}$ is perfect, $X \backslash X_{0}=\bigcup_{i \geq 1} X_{i}, \operatorname{dim}(f \triangle g)=0$ and each $g \mid X_{i}$, $i \geq 1$, is uniformly 0-dimensional (see the proof of Theorem 1.3). According to the Bogatyı theorem, there exists a sequence of 0-dimensional $G_{\delta^{-}}$ subsets $B_{k} \subset \mathbb{I}^{n}$ such that $\mathbb{I}^{n}$ is the union of any $n+1$ elements of this sequence. Let $A_{k}=(f \triangle g)^{-1}\left(Y \times B_{k}\right), k \in \mathbb{N}$. The only nontrivial condition we need to check is that each restriction $f \mid A_{k}$ is 0-dimensional, i.e. $\operatorname{dim} f^{-1}(y) \cap A_{k} \leq 0$ for all $y \in Y$ and $k \geq 1$. For fixed $y$ and $k$ we have $f^{-1}(y) \cap A_{k}=\bigcup_{i \geq 0} g_{i}^{-1}\left(B_{k}\right)$, where $g_{i}$ denotes the restriction $g \mid\left(f^{-1}(y) \cap X_{i}\right)$. Since every $g_{i}^{-1}\left(\bar{B}_{k}\right)$ is closed in $f^{-1}(y) \cap A_{k}$, it suffices to show that the sets $g_{i}^{-1}\left(B_{k}\right), i \geq 0$, are 0-dimensional. For $i=0$ this follows from the Hurewicz lowering dimension theorem [16] because $g_{0}$ is a perfect 0-dimensional map. For $i \geq 1$ we use the fact that $g \mid X_{i}$ is uniformly 0-dimensional and the preimage of any 0 -dimensional set under a uniformly 0-dimensional map is again 0-dimensional.

A map $f: X \rightarrow Y$ is said to be of countable functional weight [24] (notation $W(f) \leq \aleph_{0}$ ) if there exists a map $h: X \rightarrow Q$, where $Q$ is the Hilbert cube, such that $f \triangle h: X \rightarrow Y \times Q$ is an embedding. In [24] Pasynkov has shown that his results from [25] remain valid for maps $f$ : $X \rightarrow Y$ between finite-dimensional completely regular spaces $X$ and $Y$ such that $W(f) \leq \aleph_{0}$ and both $f$ and its Cech-Stone extension have the same dimension (the last condition holds, for example, if $X$ is normal, $Y$ paracompact and $f$ closed). We are going to show that Theorem 2.2 implies a similar result with $Y$ being a $C$-space.

Theorem 5.5. Let $f: X \rightarrow Y$ be a $\sigma$-closed $n$-dimensional surjection of countable functional weight such that $X$ is normal and $Y$ a paracompact $C$-space. Then the set $\mathcal{G}$ of all maps $g \in C\left(X, \mathbb{I}^{n}\right)$ with $\operatorname{dim}(f \triangle g)=0$ is uniformly dense in $C\left(X, \mathbb{I}^{n}\right)$. If, in addition, $X$ is paracompact and $f$ is $\sigma$-perfect, then $\mathcal{G}$ is dense and $G_{\delta}$ in $C\left(X, \mathbb{I}^{n}\right)$ with respect to the source limitation topology.

Proof. Since $W(f) \leq \aleph_{0}$, there exists a map $h: X \rightarrow Q$ such that $f \triangle h$ is an embedding. For every $k \in \mathbb{N}$ let $\gamma_{k}$ be an open cover of $Q$ of mesh $\leq k^{-1}$. Suppose $f$ is $\sigma$-closed and represent $X$ and $Y$ as the union of closed sets $X_{i}$ and $Y_{i}$, respectively, such that each $f_{i}=f \mid X_{i}$ is a closed map onto $Y_{i}$. Let $Z_{i}=\left(\beta f_{i}\right)^{-1}\left(Y_{i}\right)$ and $\bar{f}_{i}=\left(\beta f_{i}\right) \mid Z_{i}, i \in \mathbb{N}$, where $\beta f_{i}$ denotes the CechStone extension of $f_{i}$. Because $X$ is normal, each $Z_{i}$ is a closed subset of $Z=(\beta f)^{-1}(Y)$ and $\bar{f}_{i}: Z_{i} \rightarrow Y_{i}$ is a perfect $n$-dimensional map. Moreover, $Z$ is paracompact as the preimage of $Y$. We consider the extension $\bar{h}: Z \rightarrow Q$ of $h$ and the covers $\omega_{k}=\bar{h}^{-1}\left(\gamma_{k}\right) \in \operatorname{cov}(Z)$. By Theorem 2.2 (applied to the maps $\left.\bar{f}_{i}\right)$, the sets $\mathcal{H}_{i, k}$ consisting of all $g \in C\left(Z, \mathbb{I}^{n}\right)$ such that $f_{i} \triangle g$ 
is $\left(0, \omega_{k}\right)$-discrete, $i, k \in \mathbb{N}$, are open and dense in $C\left(Z, \mathbb{I}^{n}\right)$ with respect to the source limitation topology, and hence so is $\mathcal{H}=\bigcap_{i, k=1}^{\infty} \mathcal{H}_{i, k}$. Moreover, $\mathcal{H}$ is uniformly dense in $C\left(Z, \mathbb{I}^{n}\right)$. Therefore, the set $\mathcal{G}_{0}=\{g \mid X: g \in \mathcal{H}\}$ is uniformly dense in $C\left(X, \mathbb{I}^{n}\right)$. Since $h$ is a homeomorphism on every fiber of $f, \mathcal{G}_{0} \subset \mathcal{G}$. Hence, $\mathcal{G}$ is also uniformly dense in $C\left(X, \mathbb{I}^{n}\right)$.

Let $f$ be $\sigma$-perfect and $X$ paracompact. Then substituting $\bar{f}_{i}=f_{i}$, $Z_{i}=X_{i}$ and $Z=X$ in the previous proof, we deduce that $\mathcal{G}$ coincides with $\mathcal{H}$.

Corollary 5.6. Let $f: X \rightarrow Y$ be a $\sigma$-closed $n$-dimensional surjection having second countable fibers. If $X$ is metrizable and $Y$ a paracompact $C$ space, then the set of all $g \in C\left(X, \mathbb{I}^{n}\right)$ with $\operatorname{dim}(f \triangle g)=0$ is uniformly dense in $C\left(X, \mathbb{I}^{n}\right)$.

Proof. Since $f$ is of countable functional weight (see [24, Proposition 9.1]), this corollary follows from Theorem 5.5.

We finally formulate the following result; its proof is similar to that of Theorem 2.2.

TheOREM 5.7. Let $f: X \rightarrow Y$ be a perfect surjection of countable functional weight with $Y$ a paracompact $C$-space. Then all maps $g \in C(X, Q)$ such that $f \triangle g$ is an embedding form a dense $G_{\delta}$ subset of $C(X, Q)$ with respect to the source limitation topology.

Corollary 5.8. Let $f: X \rightarrow Y$ be a perfect surjection between metrizable spaces. If $Y$ is a $C$-space, then the set of all $g \in C(X, Q)$ with $f \triangle g$ being an embedding is dense and $G_{\delta}$ in $C(X, Q)$ with respect to the source limitation topology.

Addendum. In the special case when both $X$ and $Y$ are compact metric spaces, Theorem 1.3 (the existence of a map $g: X \rightarrow \mathbb{I}^{n}$ such that $\operatorname{dim}(f \triangle g)=0)$, Theorem 1.4 and Corollary 5.3 have recently been obtained by Y. Turygin in his preprint Approximation of $k$-dimensional maps. He also proved that if $f: X \rightarrow Y$ is a $k$-dimensional map between metric compacta, then the following two conditions are equivalent: (i) there exists a map $g: X \rightarrow \mathbb{I}^{k}$ with $\operatorname{dim}(f \triangle g)=0$; (ii) $f$ admits an approximation by $k$-dimensional simplicial maps (see [32] for this notion).

\section{References}

[1] D. Addis and J. Gresham, A class of infinite-dimensional spaces. Part I: Dimension theory and Alexandroff's Problem, Fund. Math. 101 (1978), 195-205.

[2] S. Bogaty̌̆, Urysohn's decomposition and Zolotarev's theorem, Vestnik Moskov. Univ. Ser. 1 Mat. 1999, no. 3, 10-14 (in Russian). 
[3] A. Chigogidze, Infinite dimensional topology and shape theory, in: Handbook of Geometric Topology, R. Daverman and R. Sher (eds.), Elsevier, Amsterdam, 2002, 307-371.

[4] - Inverse Spectra, North-Holland, Amsterdam, 1996.

[5] A. Chigogidze and V. Valov, Extensional dimension and C-spaces, Bull. London Math. Soc., to appear.

[6] S. Ditor, Averaging operators in $C(S)$ and lower semicontinuous selections of continuous maps, Trans. Amer. Math. Soc. 175 (1973), 195-208.

[7] A. Dranishnikov, The Eilenberg-Borsuk theorem for mappings into an arbitrary complex, Russian Acad. Sci. Sb. 81 (1995), 467-475.

[8] A. Dranishnikov and J. Dydak, Extension dimension and extension types, Proc. Steklov Inst. Math. 212 (1996), 55-88.

[9] A. Dranishnikov, D. Repovš and E. Ščepin, Transversal intersection formula for compacta, Topology Appl. 85 (1998), 93-117.

[10] A. Dranishnikov and V. Uspenskij, Light maps and extensional dimension, ibid. 80 (1997), 91-99.

[11] J. Dydak, Extension theory: The interface between set-theoretic and algebraic topology, Topology Appl. 74 (1996), 225-258.

[12] R. Engelking, General Topology, Heldermann, Berlin, 1989.

[13] -, Theory of Dimensions: Finite and Infinite, Heldermann, Lemgo, 1995.

[14] V. Gutev and V. Valov, Continuous selections and $C$-spaces, Proc. Amer. Math. Soc. 130 (2001), 233-242.

[15] W. Haver, A covering property for metric spaces, in: Topology Conference (Virginia Polytechnic Inst. and State Univ., 1973), R. Dickman, Jr. and P. Fletcher (eds.), Lecture Notes in Math. 375, Springer, New York, 1974, 108-113.

[16] W. Hurewicz, Über stetige Bilder von Punktmengen (Zweite Mitteilung), Proc. Akad. Amsterdam 30 (1927), 159-165.

[17] M. Katetov, On the dimension of nonseparable spaces I, Czechoslovak Math. J. 2 (77) (1952), 333-368 (in Russian).

[18] K. Kuratowski, Topology II, PWN, Warszawa, 1968.

[19] M. Levin, Bing maps and finite-dimensional maps, Fund. Math. 151 (1996), $47-52$.

[20] -, On extensional dimension of metrizable spaces, preprint.

[21] E. Michael, Continuous selections I, Ann. of Math. 63 (1956), 361-382.

[22] —, Selected selection theorems, Amer. Math. Montly 63 (1956), 233-238.

[23] J. Munkres, Topology, Prentice-Hall, Englewood Cliffs, NJ, 1975.

[24] B. Pasynkov, On geometry of continuous maps of countable functional weight, Fundam. Prikl. Mat. 4 (1998), 155-164 (in Russian).

[25] - On geometry of continuous maps of finite-dimensional compact metric spaces, Trudy Mat. Inst. Steklov. 212 (1996), 147-172 (in Russian).

[26] A. Pełczyński, Linear extensions, linear averagings, and their applications to linear topological classification of spaces of continuous functions, Dissertationes Math. 58 (1968).

[27] R. Pol, A weakly infinite-dimensional compactum which is not countable-dimensional, Proc. Amer. Math. Soc. 82 (1981), 634-636.

[28] E. Sklyarenko, A theorem on dimension-lowering mappings, Bull. Acad. Polon. Sci. Sér. Sci. Math. Astronom. Phys. 10 (1962), 429-432 (in Russian).

[29] Y. Sternfeld, On finite-dimensional maps and other maps with "small" fibers, Fund. Math. 147 (1995), 127-133. 
[30] H. Torunczyk, On CE-images of the Hilbert cube and characterization of Q-manifolds, ibid. 106 (1980), 31-40.

[31] —, Finite-to-one restrictions of continuous functions, ibid. 125 (1985), 237-249.

[32] V. Uspenskij, A selection theorem for C-spaces, Topology Appl. 85 (1998), 351-374.

Department of Mathematics

Nipissing University

100 College Drive

P.O. Box 5002

North Bay, ON, P1B 8L7, Canada

E-mail: muratt@nipissingu.ca

veskov@nipissingu.ca

Received 18 June 2001;

in revised form 16 May 2002 